\title{
Early Bone Marrow Edema Pattern of the Osteoporotic Vertebral Compression Fracture : Can Be Predictor of Vertebral Deformity Types and Prognosis?
}

\author{
Sung Eun Ahn, M.D.,, ${ }^{1,2}$ Kyung Nam Ryu, M.D., ${ }^{2}$ Ji Seon Park, M.D., ${ }^{2}$ Wook Jin, M.D., ${ }^{3}$ So Young Park, M.D., ${ }^{3}$ Sung Bum Kim, M.D. ${ }^{4}$ \\ Department of Radiology, ${ }^{1}$ Graduate School, Kyung Hee University, Seoul, Korea \\ Departments of Radiology, ${ }^{2}$ Neurosurgery, ${ }^{4}$ Kyung Hee University Hospital, Kyung Hee University School of Medicine, Seoul, Korea \\ Department of Radiology, ${ }^{3}$ Kyung Hee University Hospital at Gangdong, Kyung Hee University School of Medicine, Seoul, Korea
}

\begin{abstract}
Objective : To evaluate whether an early bone marrow edema pattern predicts vertebral deformity types and prognosis in osteoporotic vertebral compression fracture (OVCF).

Methods : This retrospective study enrolled 64 patients with 75 acute OVCFs who underwent early MRI and followed up MRI. On early MRI, the low SI pattern of OVCF on T1WI were assessed and classified into 3 types (diffuse, globular or patchy, band-like). On followed up MRI, the vertebral deformity types (anterior wedge, biconcave, crush), degree of vertebral body height loss, incidence of vertebral osteonecrosis and spinal stenosis were assessed for each vertebral fracture types.

Results : According to the early bone marrow edema pattern on T1WI, 26 vertebrae were type 1, 14 vertebrae were type 2 and 35 vertebrae were type 3. On followed up MRI, the crush-type vertebral deformity was most frequent among the type 1 OVCFs, the biconcave-type vertebral deformity was most frequent among the type 2 OVCFs and the anterior wedge-type vertebral deformity was most frequent among the type 3 OVCFs ( $p<0.001$ ). In addition, type 1 early bone marrow edema pattern of OVCF on T1WI were associated with higher incidence of severe degree vertebral body height loss, vertebral osteonecrosis and spinal stenosis on the follow up MRI.

Conclusion : Early bone marrow edema pattern of OVCF on T1WI, significant correlated with vertebral deformity types on the follow up MRI. The severe degree of vertebral height loss, vertebral osteonecrosis, and spinal stenosis were more frequent in patients with diffuse low SI pattern.
\end{abstract}

Key Words : Vertebral compression fracture $\cdot$ Osteoporosis $\cdot$ MRI $\cdot$ Bone marrow edema.

\section{INTRODUCTION}

Osteoporotic vertebral compression fracture (OVCF) is the most common fracture seen in patients with osteoporosis. The diagnosis of the OVCF may be suspected during the clinical evaluation and confirmed with radiologic findings, such as radiography, CT and MRI. Especially, the MRI is the gold standard for the investigation of vertebral bone marrow edema in OVCF. The T1WI and the fat-suppressed T2WI have been useful in detection of the faint bone marrow edema which is associated with acute $\mathrm{OVCF}^{1,2,5,8,16)}$.

The signal intensity (SI) of OVCFs differs according to the fracture ages. In the acute stage ( $<2$ months), OVCFs show low SI on T1WI and high SI on T2WI, in correspondence to the bone marrow edema and the fracture line ${ }^{19)}$. The low SI pattern of acute OVCF on T1WI can be seen as focal, band-like, or diffuse $^{7)}$. Due to fatty marrow changes of bone marrow edema, chronic OVCFs show an absence of low SI of vertebral body on T1WI otherwise ${ }^{1)}$.

Three types of vertebral deformity are usually described; wedge, biconcave, and crush deformities ${ }^{4}$. All types of deformity were associated with height loss, which was more high degree in patients with crush deformity ${ }^{4}$.

To our knowledge, there are no prior study discussed the relationship between the low SI pattern, according to early bone marrow edema pattern on T1WI and the vertebral deformity types on the follow up MRI in patients with acute OVCF. If vertebral deformity types can be predicted immediately after acute

- Received : July 22, 2015 • Revised : January 12, 2016 • Accepted : January 16, 2016

- Address for reprints : Kyung Nam Ryu, M.D.

Department of Radiology, Kyung Hee University Hospital, Kyung Hee University School of Medicine, 23 Kyungheedae-ro, Dongdaemun-gu, Seoul 02447, Korea

Tel : +82-2-958-8622, Fax : +82-2-968-0787, E-mail : t2star@naver.com

- This is an Open Access article distributed under the terms of the Creative Commons Attribution Non-Commercial License (http://creativecommons.org/licenses/by-nc/3.0) which permits unrestricted non-commercial use, distribution, and reproduction in any medium, provided the original work is properly cited. 
OVCF, more proactive treatment can be initiated relatively early. The purpose of this study was to evaluate the predictability of vertebral deformity types and prognosis using early bone marrow edema pattern of OVCF on T1WI.

\section{MATERIALS AND METHODS}

\section{Patients}

From May 2009 to January 2013, 234 patients with thoracolumbar VCF, who underwent lumbar spine MRI were retrospectively reviewed. The inclusion criteria were as followed; 1 ) patients $\geq 50$ years, 2 ) recent history of back pain after a minor trauma of less than 2 months, 3 ) lumbar bone marrow density T-score of less than $-2.5,4$ ) underwent conservative treatment, 5) patients who underwent follow up lumbar spine MRI. Exclusion criteria were as followed; 1) VCFs show absence of low SI of vertebral body on T1WI of initial MRI ( $n=104), 2)$ pathologic VCFs related to malignancy $(n=7)$ or spine infection $(n=9), 3)$ underwent surgical procedure before underment follow up MRI ( $\mathrm{n}=47)$.

Finally, 64 patients were enrolled with 75 acute OVCFs (Table 1). 10 men and 54 women with a mean age of 69.4 years (range, $56-85$ years) were included. In 5 of the 64 patients, OVCFs were diagnosed in more than one vertebral body ( 4 patients had 2 involved vertebrae and 1 patient had 3 involved vertebrae). Thirty-four fractures were in the thoracolumbar junction (T11-

Table 1. Results of osteoporotic vertebral compression fractures

\begin{tabular}{lc}
\hline \multicolumn{1}{c}{ Characteristics } & Finding \\
\hline Sex (female : male) & $5.4(54: 10)$ \\
Mean age (years) & $69.4(56-85)$ \\
Mean follow-up interval of MRI (months) & $25.77(1-98)$ \\
OVCF location & \\
T11 & $3 / 75(4 \%)$ \\
T12 & $17 / 75(22.7 \%)$ \\
L1 & $14 / 75(18.7 \%)$ \\
L2 & $11 / 75(14.7 \%)$ \\
L3 & $13 / 75(17.3 \%)$ \\
L4 & $13 / 75(17.3 \%)$ \\
L5 & $4 / 75(5.3 \%)$ \\
Low SI patterns of VB on initial MRI & \\
Type 1 & $26 / 75(34.7 \%)$ \\
Type 2 & $14 / 75(18.7 \%)$ \\
Type 3 & $35 / 75(46.7 \%)$ \\
Verebral deformity type on follow-up MRI & \\
Anterior wedge & $21 / 75(28 \%)$ \\
Biconcave & $26 / 75(34.7 \%)$ \\
Crush & $17 / 75(22.7 \%)$ \\
No change & $11 / 75(14.7 \%)$ \\
\hline
\end{tabular}

Data in parentheses are case numbers and percentage. Type $1:$ diffuse low SI (>90\% involvement), Type 2 : globular or patchy low SI (non-band like), Type 3 : band like low SI (parallel the adjacent end plate). $p<0.001$. OVCF : osteoporotic vertebral compression fracture, SI : signal intensity, VB : vertebral body
L1), and 41 fractures were in the lumbar vertebral column. The mean follow up interval of MRI was 25.7 months (range between 1-98 months).

\section{Image protocol}

Lumbar spine MRI was performed with a 1.5 Tesla (GE Medical Systems, Milwaukee, WI, USA and Siemens, Erlangen, Germany) or 3 Tesla (Achieva, Philips Medical System, the Netherlands). Axial and sagittal T1WI [repetition time (TR)/echo time (TE), 350-467/9], T2WI (TR/TE, 2750-3300/116-117) and fatsuppressed T2WI were obtained with fast spin echo techniques. Typical imaging parameters were as followed : echo train length, 20; matrix number, 384-448 $\times 256$; slice thickness, $4 \mathrm{~mm}$; field of view (FOV), 290-300 $\times 290-300 \mathrm{~mm}$ for the sagittal plane and echo train length, 14; matrix number, 320-400×256-284; slice thickness, $4 \mathrm{~mm}$; field of view (FOV), 160-180×160-180 $\mathrm{mm}$ for the axial plane.

\section{Early bone marrow edema pattern of OVCF on T1WI}

Three musculoskeletal radiologists retrospectively reviewed the lumbar spine MRIs independently and any inconsistencies were resolved by consensus. Acute OVCFs were defined as VCFs with low SI on T1WI and high SI on fat-suppressed T2WI in correspondence to the bone marrow edema, discontinuity of the vertebral cortex, or fracture line ${ }^{19)}$. The low SI pattern of OVCFs on T1WI were classified into three types. Type 1 was defined as diffuse low SI (more than $90 \%$ of vertebral body involvement), type 2 was defined as globular or patchy low SI (non-band like) and type 3 was defined as band like low SI (parallel the adjacent end plate) (Fig. 1)

\section{Vertebral deformity types, measurement of vertebral body height loss, incidence of osteonecrosis and spinal stenosis}

The vertebral deformity types of OVCFs were evaluated on the follow up MRIs. Vertebral deformity was defined as height reduction in the vertebral body with more than $20 \%$ compared with the height of adjacent vertebral bodies. The vertebral deformity types of OVCFs on follow up MRI were classified into three types based on the center of the most height reduction of vertebral body. The vertebral deformity types are classified as anterior wedge, biconcave or crush deformity depending on whether anterior, middle, or posterior portion of vertebral body was most diminished in height ${ }^{12}$. In addition, an entire height reduction with retropulsed fragment at the posterior wall of the vertebral body was defined as crush deformity (Fig. 2).

In addition, in order to determine the patient's prognosis according to the early bone marrow edema pattern on T1WI, incidence of vertebral osteonecrosis, spinal stenosis and the degree of vertebral body height loss were evaluated on the follow up MRIs. Vertebral osteonecrosis was defined as VCF with "intravertebral vacuum cleft sign" or "fluid sign"18,20,23). The intravertebral vacuum cleft sign was defined as the collection of in- 

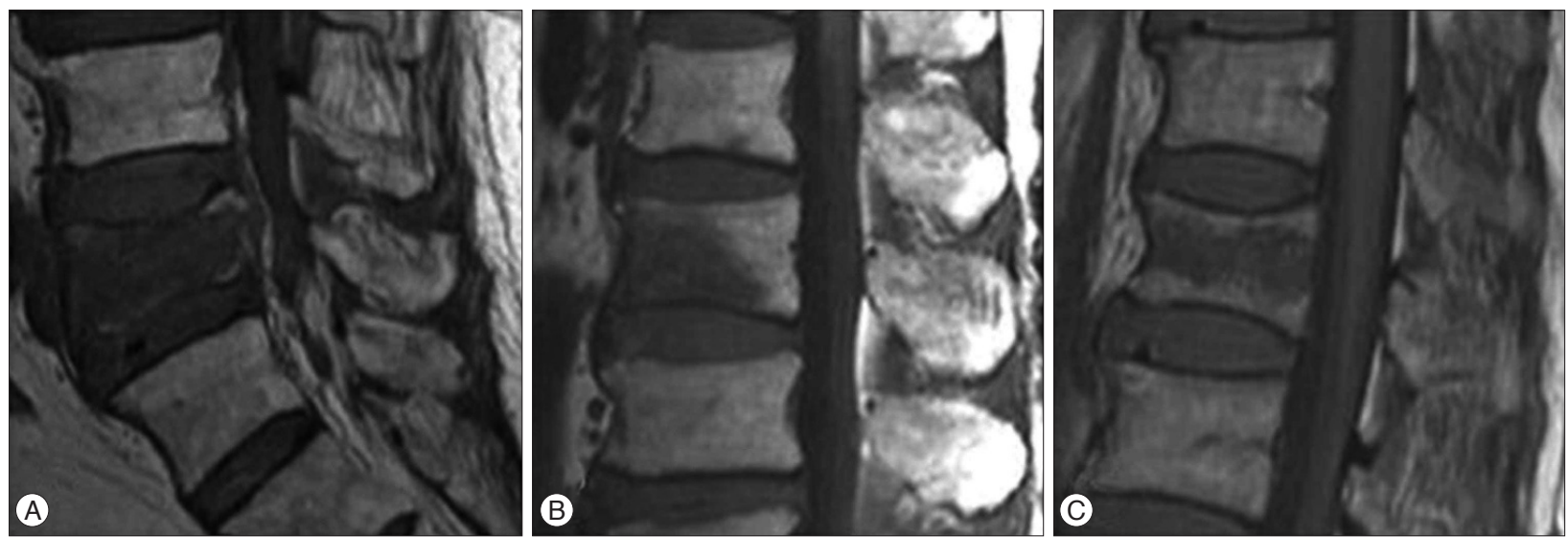

Fig. 1. The low SI types of OVCF according to the early bone marrow edema pattern on T1WI. A : Type 1 pattern with diffuse low signal intensity of VCF. B : Type 2 pattern with globular low signal intensity of VCF. C : Type 3 pattern with band-like low signal intensity of VCF. SI : signal intensity, VCF : vertebral compression fracture.
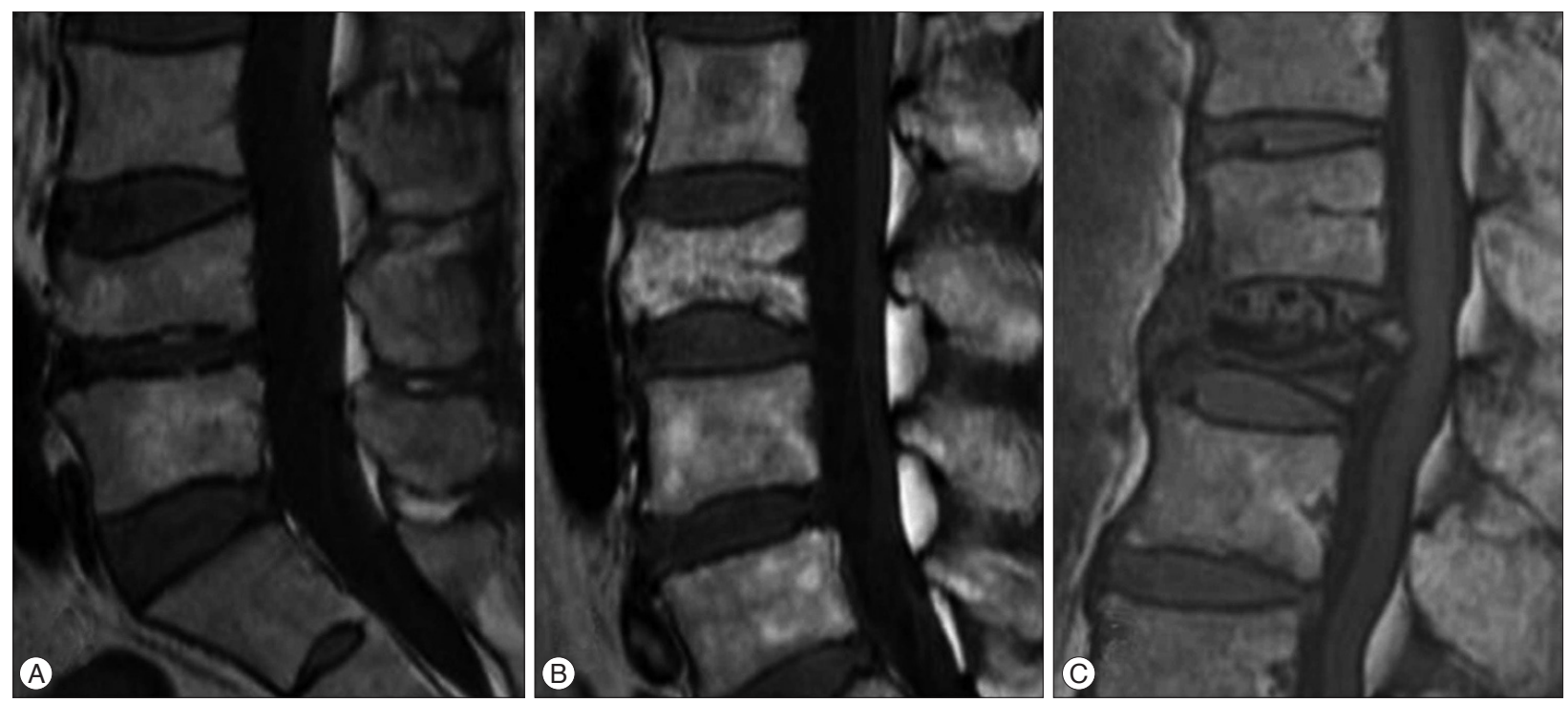

Fig. 2. The vertebral deformity types of the follow up MRI. A : Anterior wedge deformity which is defined as VB height most decline in the anterior $1 / 3$ portion of VB. B : Biconcave deformity which is defined as VB height most decline in the middle 1/3 portion of VB. C : Crush deformity which is defined as entire VB collapse with diffuse posterior bulging contour. VB : vertebral body.

travertebral air, which appears as an area of low SI on all MRI sequences. The fluid sign was defined as the collection of intravertebral fluid, which appears as an area of low SI on T1WI and high SI on T2WI, adjacent to the fractured vertebral endplate. The degree of vertebral body height loss were assessed comparing with the vertebral body height on the initial MRI. The severe degree vertebral body height loss was defined if the height reduction in the vertebral body showed more than $40 \%{ }^{12)}$.

\section{Statistical analysis}

Statistical Package for the Social Sciences (SPSS) was used for statistical analysis (SPSS 12.0, SPSS Inc., Chicago IL, USA). Frequency analysis was performed with the Fisher's exact test or ANOVA test. A $p$-value of less than 0.05 was considered to indicate a significant difference.

\section{RESULTS}

According to the early bone marrow edema pattern on T1WI, 26 (34.6\%) showed type 1 pattern, 14 (18.7\%) showed type 2 pattern and $35(46.7 \%)$ showed type 3 pattern. On the follow up MRI, 21 (28\%) showed an anterior wedge deformity, 26 (34.7\%) showed a biconcave deformity and 17 (22.7\%) showed a crush deformity. 11 (14.7\%) OVCFs showed a height reduction less than $20 \%$ in the vertebral body (Table 1 ).

The mean age was 73.5 years in type 1 group, 66.3 years in the type 2 group, and 68.8 years in the type 3 group. The difference was statistical significant $(p<0.005)$. Among 26 patients of type 1 group, 5 patients (19.23\%) who the conservative management failed, underwent surgical intervention (3 patients : laminectomy with posterior instrumentation, 1 patient : laminectomy, 1 patients : partial corpectomy and cylinder insertion with posterior 
Table 2. The relationships between early bone marrow edema pattern on T1WI and vertebral deformity types on follow-up MRI

\begin{tabular}{lccc}
\hline \multicolumn{1}{c}{$\begin{array}{c}\text { Vertebral deformity types } \\
\text { on follow-up MRI }\end{array}$} & \multicolumn{3}{c}{ Low SI types of OVCF on T1WI } \\
\cline { 2 - 4 } \multicolumn{1}{c}{ Type 1 $(\mathrm{n}=26)$} & Type 2 (n=14) & Type 3 (n=35) \\
\hline Anterior wedge deformity $(\mathrm{n}=21)$ & $3 / 26(11.5 \%)$ & $1 / 14(7.1 \%)$ & $7 / 35(48.6 \%)$ \\
Biconcave deformity $(\mathrm{n}=26)$ & $9 / 26(34.6 \%)$ & $10 / 14(71.4 \%)$ & $3 / 35(8.6 \%)$ \\
Crush deformity $(\mathrm{n}=17)$ & $13 / 26(50 \%)$ & $1 / 14(7.1 \%)$ & $8 / 35(22.9 \%)$ \\
No change of VB configuration $(\mathrm{n}=11)$ & $1 / 26(3.8 \%)$ & $2 / 14(14.3 \%)$ & \\
\hline
\end{tabular}

Data in parentheses are case numbers and percentage, $p<0.001$. Type 1 : diffuse low SI ( $>90 \%$ involvement), Type $2:$ globular or patchy low SI (non-band like), Type 3 : band like low SI (parallel the adjacent end plate). SI : signal intensity, VB : vertebral body

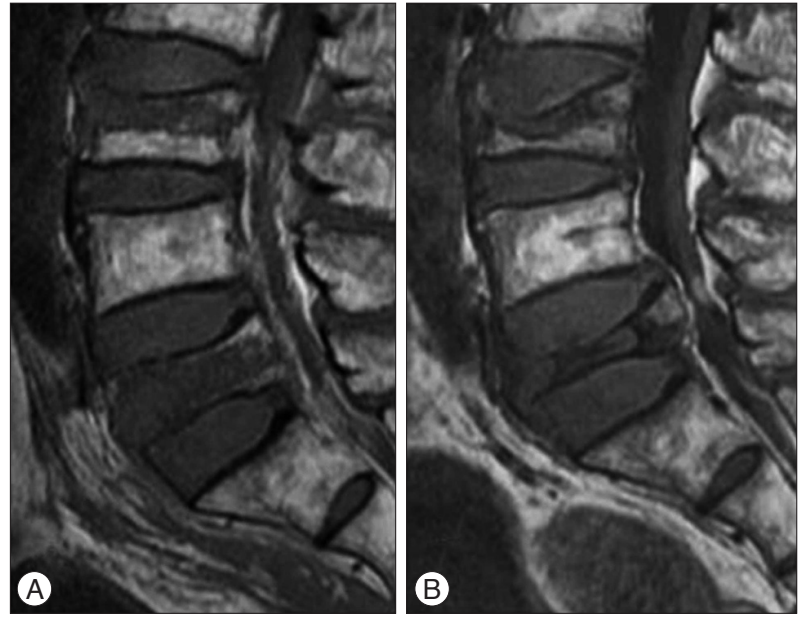

Fig. 3. A 77-year-old man with OVCFs at the L3 and L5 vertebral bodies. A : Sagittal T1WI of initial MRI shows band-like low signal intensity (type 3 pattern) of VCF at the L3 vertebral body and diffuse low signal intensity (type 1 pattern) of VCF at the L5 vertebral body. B : Sagittal T1WI of follow up MRI taken after 4 months shows anterior wedge deformity of VCF at the L3 vertebral body and crush deformity of VCF at the L5 vertebral body. L5 vertebral body exhibits severe degree vertebral body height loss (>40\%) and posterior bulging with spinal stenosis. He failed conservative management and underwent surgical treatment (laminectomy with posterior instrumentation). OVCFs : osteoporotic vertebral compression fractures, VCF : vertebral compression fracture.

instrumentation). All 14 patients of type 2 group were managed with conservative treatments. Among 35 patients of type 3 group, 5 patients (14.29\%) who the conservative management failed, underwent surgical intervention (laminectomy with posterior instrumentation).

The results of the relationship between the low SI of OVCFs according to early bone marrow edema pattern on T1WI and vertebral deformity types of OVCFs on the follow up MRI are shown in Table 2. For each low SI type according to the early bone marrow edema pattern on T1WI, the frequency of vertebral deformity type was assessed. Among 26 OVCFs with type 1 low SI pattern, 3 OVCFs (11.5\%) showed anterior wedge deformity, 9 OVCFs (34.6\%) showed biconcave deformity and 13 OVCFs (50\%) showed crush deformity on the follow up MRI (Fig. 3). 1 OVCF (3.8\%) showed no change of vertebral body configuration. Among 14 OVCFs with type 2 low SI pattern, 1 OVCF (7.1\%) showed anterior wedge deformity, 10 OVCFs (71.4\%) showed biconcave deformity (Fig. 4, 5) and 1 OVCF (7.1\%) showed crush deformity on the follow up MRI. 2 OVCFS (14.3\%) showed no
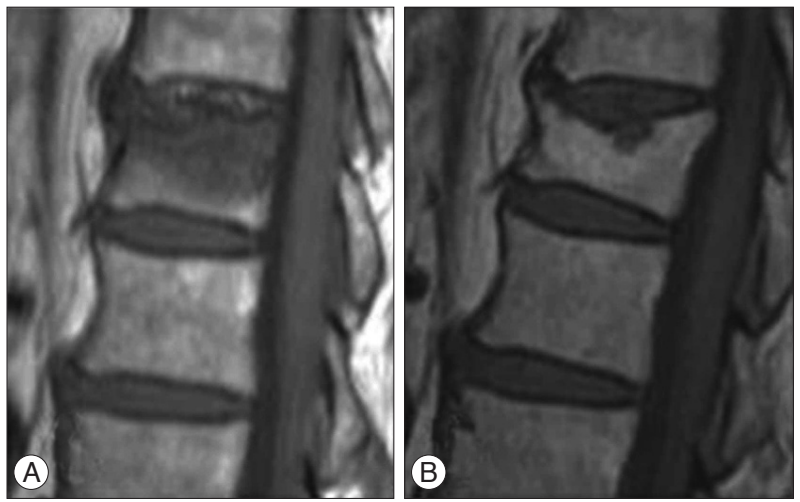

Fig. 4. 67-year-old woman with OVCF of the T12 vertebral body. A : Sagittal T1WI of initial MRI shows acute VCF of the superior T12 endplate with globular low signal intensity (type 2 pattern). B : Sagittal T1WI of follow up MRI taken after 61 months shows chronic VCF of the T12 vertebral body with biconcave deformity. Note the chronic inflammatory changes and fatty infiltration of bone marrow below fractured superior end plate. Note the severe degree vertebral body height loss $(>40 \%)$. OVCFs : osteoporotic vertebral compression fractures, VCF : vertebral compression fracture.
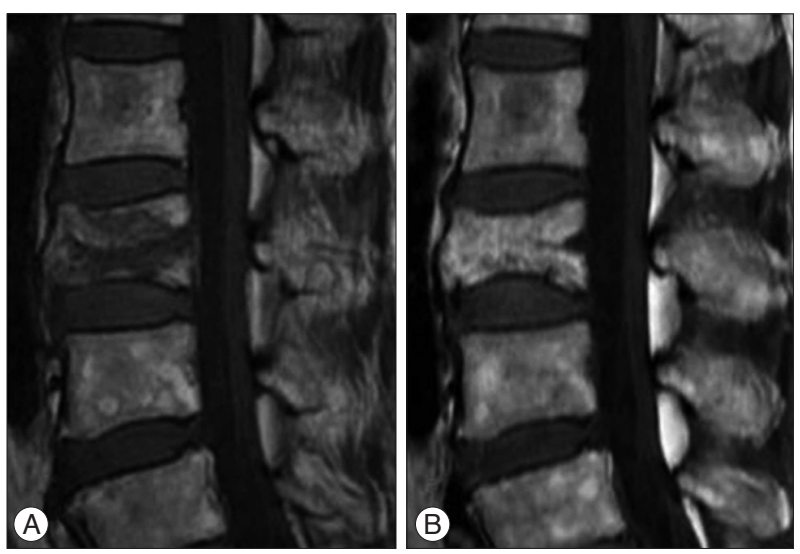

Fig. 5. 61-year-old woman with OVCF of the L3 vertebral body. A : Sagittal T1WI of initial MRI shows acute VCF of the L3 vertebral body with patchy low signal intensity (type 2 pattern). B : Sagittal T1WI of follow up MRI taken after 5 months shows chronic VCF of the L3 vertebral body with biconcave deformity. Note the mild degree vertebral body height loss. OVCFs : osteoporotic vertebral compression fractures, VCF : vertebral compression fracture.

changes of the vertebral body configuration. Among 35 OVCFs with type 3 low SI pattern, 17 OVCFs (48.6\%) showed anterior wedge deformity (Fig. 3), 7 OVCFs (20\%) showed biconcave deformity and 3 OVCFs (8.6\%) showed crush deformity on the follow up MRI. 8 OVCFs (22.9\%) showed no change of the verte- 
Table 3. The relationships between low SI patterns of OVCF according to the early T1-weighted MRI-based classification and risk factors

\begin{tabular}{lcccc}
\hline \multirow{2}{*}{ Risk factors } & \multicolumn{3}{c}{ Low SI types of OVCF on T1WI } & p-value \\
\cline { 2 - 4 } & Type 1 $(\mathrm{n}=26)$ & Type 2 $(\mathrm{n}=14)$ & Type 3 $(\mathrm{n}=35)$ & 0.113 \\
Mean VB height loss on f/u MRI & $39.31 \%$ & $31.71 \%$ & $29.24 \%$ & $<.001$ \\
Severe (>40\%) VB height loss on f/u MRI & $14 / 26(53.9 \%)$ & $4 / 14(28.8 \%)$ & $13 / 35(37.1 \%)$ & $<0.001$ \\
Vertebral osteonecrosis & $9 / 26(34.6 \%)$ & $1 / 14(7.1 \%)$ & $2 / 35(5.7 \%)$ & $<0.001$ \\
Spinal stenosis & $16 / 26(61.5 \%)$ & $1 / 14(7.1 \%)$ & $6 / 35(17.1 \%)$ & \\
\hline
\end{tabular}

Data in parentheses are case numbers and percentage. Type $1:$ diffuse low SI (>90\% involvement), Type $2:$ globular or patchy low SI (non-band like), Type $3:$ band like low SI (parallel the adjacent end plate). SI : signal intensity, VB : vertebral body, f/u : follow up

bral body configuration. In summary, differences of the vertebral deformity types of OVCFs were visible on the follow up MRIs, according to the early bone marrow edema pattern on T1WI. Crush deformities were more frequent in patients with type 1 low SI pattern on T1WI (13/26, 50\%), biconcave deformities were more frequent in patients with type 2 low SI pattern (10/14, $71.4 \%$ ) and anterior wedge deformities were more frequent in patients with type 3 low SI pattern (17/35, 48.6\%). The difference was statistical significant $(p<0.001)$.

The results of the patient's prognosis according to early T1weighted MRI-based classification are shown in Table 3. The mean vertebral body height loss was $39.3 \%$ in the type 1 group, $31.7 \%$ in the type 2 group, and $29.2 \%$ in the type 3 group. The mean vertebral body height loss was higher in type 1 bone marrow edema pattern on T1WI, but the difference was not statistical significant ( $p=0.113$ ). However, the incidence of severe degree vertebral body height loss (height reduction more than $40 \%$ ) was significantly higher in the type 1 group (53.9\%) than in type 2 group (28.6\%) or type 3 group (37.1\%).

Also the incidence of vertebral osteonecrosis and spinal stenosis was significantly higher in the type 1 group than in others. Among 26 subjects of the type 1 group, 9 (34.6\%) showed vertebral osteonecrosis, among 14 subjects of the type 2 group, 1 (7.1\%) showed and among 35 subjects of the type 3 group, 2 (5.7\%) showed. The difference was statistical significant $(p<0.001)$.

Among 26 subjects of the type 1 group, 16 (61.5\%) showed spinal stenosis, among 14 subjects of the type 2 group, 1 (7.1\%) showed and among 35 subjects of the type 3 group, 6 (17.1\%) showed. The difference was statistical significant $(\mathrm{p}<0.001)$.

\section{DISCUSSION}

The diagnosis of the OVCF may be suspected during the clinical evaluation and confirmed with radiologic findings such as radiography, CT and MRI. Morphologic features of OVCFs include the height reduction in vertebral bodies and various vertebral body deformities on plain radiographies. But due to inadequate film quality false-negative rates of plain radiography from $27 \%$ to $45 \%$ were reported in previous studies ${ }^{12)}$. The CT is one of the most suitable imaging techniques for the evaluation of bone structures can be used to establish the degree of cortical bone destruction. The MRI is considered as useful for the differentiation between chronic and acute fractures and for early diag- nosis especially, because the bone marrow edema which are associated with fractures of the vertebral body are clearly shown as SI changes.

Patients with OVCFs were managed with conservative treatments, including pain control, short period of bed rest and a brace regularly. The most of the patients showed a successful pain relief 3 months after the fracture ${ }^{9,21)}$. Some patients who the conservative management failed and the pain continued because of a severe degree vertebral body height loss or kyphosis may be candidates for invasive therapies such a vertebroplasty, balloon kyphoplasty and posterior lumbar interbody fusion (PLIF). The patients with OVCF that have $>40 \%$ loss of vertebral height loss, or $>30$ degrees of kyphosis often can be treated with surgical intervention $^{15}$. However no obvious consensus has been reached yet about the surgical indications in the OVCFs treatment with the after failure of conservative management exemption. Also numerous controversies exist about the time point of surgical treatment ${ }^{3,6,10,11,13,17)}$. Therefore if vertebral deformity types and degrees of OVCF can be predicted at early stage, more active treatment can be initiated relatively early.

In this study acute OVCFs were classified into three types of low SI pattern according to the early bone marrow edema pattern on T1WI and the relationship between the low SI pattern and final vertebral deformity types of OVCFs on follow up MRI were assessed on the follow. Differences of the final vertebral deformity types of OVCFs according to the early bone marrow edema pattern on T1WI were statistical significant $(p<0.001)$. Especially, when the acute OVCFs showed diffuse low SI on T1WI, the most frequent vertebral deformity type on the follow up MRI was the posterior portion of vertebral body height loss or an entire height reduction with retropulsed fragment at the posterior wall of the vertebral body.

In addition, in the present study, the severe degree vertebral body height loss (height reduction more than 40\%) was more frequent in patients with diffuse low SI pattern on T1WI of initial MRI. According to previous study, higher rates of initial vertebral collapse (more than 28.5\%) are known as risk factors for conservative treatment failures in patients with $\mathrm{OVCF}^{10}$.

Also, associated vertebral osteonecrosis and spinal stenosis was more frequent in patients with diffuse low SI pattern on T1WI of initial MRI. These results were similar to the results of a previous study reported by Kanchiku at al. ${ }^{7}$, where the relationship between SI patterns of OVCFs based on MRI and pa- 
tient's outcome were examined. In that study the signal changes of OVCFs were classified into six types based on T1WI in the center of the vertebral bodies (total, anterior, posterior, superior, inferior, and central). There was found no intraspinal protrusion in the inferior and superior types and the neurological stability was achieved but otherwise there was found a high frequency of intraspinal protrusion in the total and posterior types. And OVCFs associated with vertebral osteonecrosis were known for their poor prognosis ${ }^{14,18,20,22,23)}$. Therefore, for subjects with acute OVCFs, especially with diffuse low SI pattern on T1WI, an appropriate treatment based on a MRI diagnosis of low SI pattern early after the injury should be concerned to prevent the progression of the vertebral deformity or the affected vertebral body to a severe degree vertebral collapse.

The present study had several limitations. Like in the most retrospective studies, the MRI follow up periods were not uniform for each patient. In addition, we only included patients who underwent conservative treatment and underwent follow up MRI. A further limitation was the number of type 2 subjects compared to the numbers of type 1 or type 3 subjects. But to our knowledge, this report is the first study for a description of the relationship between the early bone marrow edema pattern on TIWI and the vertebral deformity types on follow up MRI. It is also the first study for a prognosis evaluation for patients under the use of early bone marrow edema pattern on T1WI.

\section{CONCLUSION}

In conclusion, early bone marrow edema pattern of OVCF on T1WI, significant correlated with vertebral deformity types on the follow up MRI $(p<0.001)$. Poor prognostic factors such as higher rates of vertebral body height loss, crush deformity and severe degree vertebral body height loss (height reduction more than $40 \%$ ) on the follow up MRI were more frequent in patients with diffuse low SI type according to the early bone marrow edema pattern on T1WI. In addition, vertebral osteonecrosis and spinal stenosis on the follow up MRI were more frequent in patients with diffuse low SI type. Therefore the diffuse low SI type according to the early bone marrow edema pattern on TIWI can be considered as one of the poor prognostic factors for the acute OVCFs.

\section{References}

1. Baker LL, Goodman SB, Perkash I, Lane B, Enzmann DR : Benign versus pathologic compression fractures of vertebral bodies : assessment with conventional spin-echo, chemical-shift, and STIR MR imaging. Radiology 174 : 495-502, 1990

2. Baur A, Stäbler A, Arbogast S, Duerr HR, Bartl R, Reiser M : Acute osteoporotic and neoplastic vertebral compression fractures : fluid sign at MR imaging. Radiology 225 : 730-735, 2002

3. Buchbinder R, Osborne RH, Ebeling PR, Wark JD, Mitchell P, Wriedt C, et al. : A randomized trial of vertebroplasty for painful osteoporotic vertebral fractures. N Engl J Med 361 : 557-568, 2009

4. Ismail AA, Cooper C, Felsenberg D, Varlow J, Kanis JA, Silman AJ, et al. :
Number and type of vertebral deformities : epidemiological characteristics and relation to back pain and height loss. European Vertebral Osteoporosis Study Group. Osteoporos Int 9 : 206-213, 1999

5. Jung HS, Jee WH, McCauley TR, Ha KY, Choi KH : Discrimination of metastatic from acute osteoporotic compression spinal fractures with MR imaging. Radiographics $23: 179-187,2003$

6. Kallmes DF, Comstock BA, Heagerty PJ, Turner JA, Wilson DJ, Diamond $\mathrm{TH}$, et al. : A randomized trial of vertebroplasty for osteoporotic spinal fractures. N Engl J Med 361 : 569-579, 2009

7. Kanchiku T, Taguchi T, Kawai S : Magnetic resonance imaging diagnosis and new classification of the osteoporotic vertebral fracture. J Orthop Sci $8:$ 463-466, 2003

8. Kazawa N : T2WI MRI and MRI-MDCT correlations of the osteoporotic vertebral compressive fractures. Eur J Radiol 81 : 1630-1636, 2012

9. Kim DH, Vaccaro AR: Osteoporotic compression fractures of the spine; current options and considerations for treatment. Spine J $6: 479-487$, 2006

10. Lee HM, Park SY, Lee SH, Suh SW, Hong JY : Comparative analysis of clinical outcomes in patients with osteoporotic vertebral compression fractures (OVCFs) : conservative treatment versus balloon kyphoplasty. Spine J 12 : 998-1005, 2012

11. Lee SG, Yoo CJ : Percutaneous vertebroplasty in the treatment of vertebral body compression fracture with osteoporosis : preliminary report. J Korean Neurosurg Soc 29: 615-622, 2000

12. Lenchik L, Rogers LF, Delmas PD, Genant HK : Diagnosis of osteoporotic vertebral fractures : importance of recognition and description by radiologists. AJR Am J Roentgenol 183 : 949-958, 2004

13. Liu JT, Liao WJ, Tan WC, Lee JK, Liu CH, Chen YH, et al. : Balloon kyphoplasty versus vertebroplasty for treatment of osteoporotic vertebral compression fracture : a prospective, comparative, and randomized clinical study. Osteoporos Int 21 : 359-364, 2010

14. McKiernan F, Faciszewski T : Intravertebral clefts in osteoporotic vertebral compression fractures. Arthritis Rheum 48 : 1414-1419, 2003

15. Mikles MR, Stchur RP, Graziano GP : Posterior instrumentation for thoracolumbar fractures. J Am Acad Orthop Surg 12 : 424-435, 2004

16. Oner FC, van Gils AP, Dhert WJ, Verbout AJ : MRI findings of thoracolumbar spine fractures : a categorisation based on MRI examinations of 100 fractures. Skeletal Radiol 28 : 433-443, 1999

17. Robinson Y, Olerud C : Vertebroplasty and kyphoplasty--a systematic review of cement augmentation techniques for osteoporotic vertebral compression fractures compared to standard medical therapy. Maturitas $72: 42-49,2012$

18. Ryu CW, Han H, Lee YM, Lim MK : The intravertebral cleft in benign vertebral compression fracture : the diagnostic performance of non-enhanced MRI and fat-suppressed contrast-enhanced MRI. Br J Radiol 82 : 976-981, 2009

19. Ryu KN, Jin W, Ko YT, Yoon Y, Oh JH, Park YK, et al. : Bone bruises : MR characteristics and histological correlation in the young pig. Clin Imaging $24: 371-380,2000$

20. Theodorou DJ : The intravertebral vacuum cleft sign. Radiology 221 : 787-788, 2001

21. Venmans A, Klazen CA, Lohle PN, Mali WP, van Rooij WJ : Natural history of pain in patients with conservatively treated osteoporotic vertebral compression fractures : results from VERTOS II. AJNR Am J Neuroradiol 33 : 519-521, 2012

22. Wiggins MC, Sehizadeh M, Pilgram TK, Gilula LA : Importance of intravertebral fracture clefts in vertebroplasty outcome. AJR Am J Roentgenol 188 : 634-640, 2007

23. Yu CW, Hsu CY, Shih TT, Chen BB, Fu CJ : Vertebral osteonecrosis : MR imaging findings and related changes on adjacent levels. AJNR Am J Neuroradiol $28: 42-47,2007$ 